\title{
Proposed Biology of the Spontaneous Remission of Hepatocellular Carcinoma
}

\author{
Behzad Niakan* \\ Pharm. D, Los Angeles, CA, USA
}

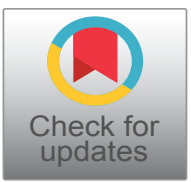

*Corresponding author: Behzad Niakan, Pharm. D., P.O. Box 25817, Los Angeles, Ca. 90025, USA, Tel: (310)-925-2720

\begin{abstract}
Case Reports of the Spontaneous regression or remission of hepatocellular carcinoma was studied. Two common factors were noted among the case reports of the prolonged spontaneous remission or regression of hepatocellular carcinoma. Low blood oxygen and a short and steady or rapid growth of hepatocellular carcinoma before the spontaneous remission or regression of hepatocellular carcinoma. It is suggested that the rapid growth of the hepatocellular carcinoma faced with low blood oxygen has possibly resulted in the hepatocellular carcinoma becoming vulnerable to an immune response. The rapid growth of the primary tumor not the growth of new metastases.
\end{abstract}

\section{Introduction}

Hepatocellular carcinoma is an aggressively growing tumor. Case reports of Spontaneous disappearance of hepatocellular carcinoma that had been verified (pathology, blood test, etc.) without any medical intervention has baffled many researchers. It may regress for a short time and return. It may disappear for a prolonged period without any signs or symptoms. Two events preceding the prolonged spontaneous remission of hepatocellular carcinoma seem to be a low blood oxygen and a short and steady or rapid growth of hepatocellular carcinoma just before the spontaneous regression or remission of hepatocellular carcinoma.

\section{Methods}

Reports of spontaneous remission and regression of hepatocellular carcinoma in the medical literature was studied. Mostly the English medical literature was referenced. The case reports of the spontaneous regression or remission of hepatocellular carcinoma were separated into short or temporary regression or a prolonged remission. The case reports of prolonged spontaneous remission of hepatocellular carcinoma were classified by the event preceding the spontaneous regression or remission of hepatocellular carcinoma $A$ common denominator was sought among them.

\section{Results}

Two common factors among the case reports of prolonged spontaneous regression or remission of hepatocellular carcinoma were identified. The authors of the case report had noted a steady or rapid growth just prior to the spontaneous regression or remission of hepatocellular carcinoma [1-5]. Secondly, a low blood oxygen just before the spontaneous regression or remission of hepatocellular carcinoma was noted in the reported cases.

Case reports of the prolonged Spontaneous regression or remission of hepatocellular carcinoma more commonly occur after tumor hypoxia (occlusion of the hepatic artery or portal Vein) [6], hepatitis and liver cirrhosis [1,7-9] liver cirrhosis [2,10,11], hemorrhage $[1,12,13]$, being on dialysis [14-16], lung metastases [17-19] all have in common a low blood oxygen delivery to the tumor. Occlusion of hepatic artery or the portal vein may result in a reduced or blockage of blood to the tumor, liver cirrhosis may result in macrocytic anemia, hepatitis may result in liver cirrhosis, hemorrhage may result in low blood oxygen transport, dialysis may reduce red blood cell and low kidney function may result in a lower production of erythropoietin thereby a lower red cell count. Pulmonary metastases may reduce blood oxygen supply (Table 1).

A case report of the spontaneous regression of 
Table 1: Spontaneous Regression or Remission Hepatocellular Carcinoma.

\begin{tabular}{|c|c|c|c|c|c|c|c|}
\hline & $\begin{array}{l}\text { Rapid } \\
\text { Growth }\end{array}$ & $\begin{array}{l}\text { Tumor } \\
\text { Embolism }\end{array}$ & $\begin{array}{l}\text { Liver } \\
\text { Cirrhosis }\end{array}$ & $\begin{array}{l}\text { Lung } \\
\text { Metastases }\end{array}$ & $\begin{array}{l}\text { Renal } \\
\text { Dialysis }\end{array}$ & Hemorrhage & Hepatitis \\
\hline Ref. \# 1 & $x$ & & $x$ & & & $x$ & $x$ \\
\hline Ref. \# 2 & $x$ & & $x$ & & & & \\
\hline Ref. \# 3 & $x$ & & & & & & $x$ \\
\hline Ref. \# 4 & $x$ & & $x$ & & & & \\
\hline Ref. \# 5 & $x$ & & $x$ & & & & \\
\hline Ref. \# 6 & & $x$ & & & & & \\
\hline Ref. \# 7 & & & $x$ & & & & \\
\hline Ref. \# 8 & & & $x$ & & & & \\
\hline Ref. \# 9 & & $x$ & $x$ & $x$ & & & $x$ \\
\hline Ref. \# 10 & & & $x$ & & & & \\
\hline Ref. \# 11 & & & $x$ & & & & \\
\hline Ref. \# 12 & & & $x$ & & & $x$ & \\
\hline Ref. \# 13 & & & $x$ & & & $x$ & \\
\hline \multicolumn{8}{|l|}{ Ref. \# 14} \\
\hline Ref. \# 15 & & & & $x$ & $x$ & & \\
\hline Ref. \# 16 & & & & & $x$ & & $x$ \\
\hline Ref. \# 17 & & & & $x$ & & & $x$ \\
\hline Ref. \# 18 & & & & $x$ & & & \\
\hline Ref. \# 19 & & & & $x$ & & & $x$ \\
\hline
\end{tabular}

hepatocellular carcinoma is noteworthy [20]. A patient with hepatocellular carcinoma had a cerebrovascular accident and was placed on enalapril, furosemide and Curcumin (most active ingredient of Turmeric). Afterwards the hepatocellular carcinoma disappeared. Cerebrovascular accident may result in pulmonary edema. Furosemide is a diuretic possibly the patient had pulmonary edema. Curcumin is an iron chelator and forms complex solubilized with up to $90 \%$ of blood iron. Therefore, low blood oxygen.

\section{Discussion}

It appears the prolonged spontaneous regression or remission of hepatocellular carcinoma is often associated with a low blood oxygen and an aggressively growing hepatocellular carcinoma. One may speculate of its significance.

Possibly an aggressively growing hepatocellular carcinoma faced with a low blood oxygen supply has resulted in a loss of hemostasis within the malignant growth. Possibly the balance between the well oxygenated area and hypoxia area within the tumor is disrupted. The primary tumor becoming non-viable and being removed by the immune system. Thereafter, immunity being developed against the hepatocellular carcinoma.

Liver cirrhosis may result in hepatocellular carcinoma and anemia. An aggressively growing hepatocellular carcinoma faced with low blood oxygen may regress spontaneously.
In a paper published by Jonathan I Huz, et al. [6] "spontaneous regression of hepatocellular carcinoma is most often associated with tumor hypoxia or a systemic inflammatory response" the authors noted a correlation between spontaneous regression of hepatocellular carcinoma with hypoxia and systemic inflammation. This author would like to suggest tumor hypoxia in the presence of a short and steady or rapid growth of hepatocellular carcinoma possibly resulted in a spontaneous regression of hepatocellular carcinoma.

Steady or rapid tumor growth and low blood oxygen just prior to the spontaneous remission of hepatocellular carcinoma is not specific to hepatocellular carcinoma. Reports of spontaneous remissions of other types of cancer such as renal cell carcinoma are also preceded by a steady or rapid growth and a low blood oxygen just prior to spontaneous regression or remission "proposed biology of the spontaneous remission of cancer [22].

It is noteworthy that spontaneous remission of cancer in rat may occur if the tumor grows steadily and faced by a low blood oxygen level.

The paper published by Manon Buijs, et al. [21], Indicated that N1S1 cells and McA-RH7777 cells were implanted in the liver of Sprague-Dawley rats. Tumor growth continued to a size and afterwards regressed and complete regression of all tumors were seen five to six weeks after tumor implantation. According to the authors tumors grew to relatively large size compared to the overall size of the liver in rats. Possibly the increase in tumor size has resulted in an increase in 
blood volume and the lungs delivering the same amount of oxygen has resulted in a drop in blood oxygen level. Also, liver tumor may concentrate in iron and reduce blood oxygen delivery from lungs. Therefore, a steady growth of a tumor faced with an increasing lower blood oxygen level.

\section{Caution}

A hypothesis has been suggested without any experimental proof for its correctness. Medical supervision required for any experimental treatment. Experimental treatment may result in oxygen levels dropping too low. Inducing tumor growth may endanger a vital organ or cause an irreversible damage or may cause tumor to become more aggressive, etc.

\section{Disclaimer}

Always seek the advice of your physician or other qualified health provider with any questions you may have regarding a medical condition. Never disregard professional medical advice or delay in seeking it because you have read this study. This is not intended to be a substitute for medical advice, diagnosis, or treatment in regard to any patient.

\section{References}

1. Kondo S, Okusaka T, Ueno H, Ikeda M, Morizane C (2006) Spontaneous regression of hepatocellular carcinoma. Int $J$ Clin Oncol 11: 407-411.

2. Suzuki M, Okazaki N, Yoshino M, Yoshida T (1989) Spontaneous regression of hepatocellular carcinoma-a case report. Hepatogastroenterology 36: 160-163.

3. Matsuo $R$, Ogata $H$, Tsuji $H$, Kitazono $T$, Shimada $M$, et al. (2001) Spontaneous regression of hepatocellular carcinoma-a case report. Hepatogastroenterology 48: 1740-1742.

4. Nakayama S (2012) Spontaneous regression of hepatocellular carcinoma. Indian journal of gastroenterology 31: $267-270$.

5. Iwasaki M, Furuse J, Yoshino M, Moriyama N, Kanemoto $\mathrm{H}$, et al. (1997) Spontaneous regression of hepatocellular carcinoma: a case report. Jpn J Clin Oncol 27: 278-281.

6. Huz JI, Melis M, Sarpel U (2012) Spontaneous regression of hepatocellular carcinoma is most often associated with tumor hypoxia or a systemic inflammatory response. HPB (oxford) 14: 500-505.

7. Meza-Junco J, Montaño-Loza AJ, Martinez-Benítez B, Cabrera-Aleksandrova T (2007) Spontaneous partial regression of hepatocellular carcinoma in a cirrhotic patient. Ann Hepatol 6: 66-69.
8. Chien RN, et al. (1992) Spontaneous regression of hepatocellular carcinoma.Am J Gastroenterol 87: 903-905.

9. Pectasides E, et al. (2016) Spontaneous regression of hepatocellular carcinoma with multiple lung metastases: A case report and review of the literature. Dig Dis Sci 61: $2749-2754$

10. Martin EF, Huang M, Madrazo B, Levy C (2014) Spontaneous regression hepatocellular carcinoma after eradication of HCV. Journal of Gastroenterology, Pancreatology \& Liver Disorders 1: 1-4

11. Li A-J, Wu MC, Cong WM, Shen F, Yi B (2003) Spontaneous complete necrosis of hepatocellular carcinoma: A case report. Hepatobiliary Pancreat Dis Int 2: 152-154.

12. Tocci G, Conte A, Guarascio P, Visco G (1990) Spontaneous remission of hepatocellular carcinoma after massive gastrointestinal haemorrhage. BMJ 300: 641-642.

13. Sato Y, Fujiwara K, Nakagawa S, Kanishima S, Ohta Y, et al. (1985) A case of spontaneous regression of hepatocellular carcinoma with bone metastasis. Cancer 56: 667-671.

14. Muroya D, Sato T, Sakai H, Hisaka T, Akagi $Y$, et al. (2021) Spontaneous regression of lung metastases in hepatocellular carcinoma: a case report. Int J Surg Case Rep 78: 378-381.

15. Harimoto N, Shirabe K, Kajiyama K, Gion T, Takenaka M, et al. (2012) Spontaneous regression of multiple pulmonary recurrences of hepatocellular carcinoma after hepatectomy: Report a case. Surg Today 42: 475-478.

16. Sakamaki A, Kamimura K, Abe S, Tsuchiya A, Takamura M, et al. (2017) Spontaneous regression of hepatocellular carcinoma: A mini review. World J Gastroenterol 23: 37973804.

17. Saito T, Naito M, Matsumura $Y$, Kita H, Kanno T, et al. (2014) Spontaneous regression of a large hepatocellular carcinoma with multiple lung metastases. Gut Liver 8: 569574.

18. Gunasekaran SS, Emmadi R, Landers LA, Gaba RC (2016) Regression of hepatocellular carcinoma lung metastases after guyabano fruit extract consumption. J Diet Suppl 13: 237-244.

19. Lim DH, Park KW, Lee SI (2014) Spontaneous complete regression of multiple metastases of hepatocellular carcinoma: a case report. Oncology Lett 7: 1225-1228.

20. Noij DP, van Der Linden PWG (2016) Spontaneous regression of hepatocellular carcinoma in a Caucasian male patient: a case report and review of the literature. Mol Clin Oncol 6: 225-228.

21. Buijis M, Geschwind JFH, Syed LH, Ganapathy-Kanniappan S, Kunjithapatham R, et al. (2012) Spontaneous regression in a syngeneic rat model of liver cancer: implications for survival studies. J Vasc Interv Radiol 23: 1685-1691.

22. Niakan B (2021) Proposed Biology of the Spontaneous Remission of Cancer. Int J Cancer Clin Res 8: 158. 